Polymer Journal, Vol. 38, No. 10, pp. 1081-1088 (2006)

(C) 2006 The Society of Polymer Science, Japan

\title{
Dielectric Normal Mode Relaxation of Poly(propylene oxide) and Poly(butylene oxide) in Dilute and Semidilute Solutions
}

\author{
Hiroshi Nishimura, Osamu URAKAwA, and Keiichiro ADACHI ${ }^{\dagger}$ \\ Department of Macromolecular Science, Graduate School of Science, \\ Osaka University, Toyonaka 560-0043, Japan
}

(Received June 22, 2006; Accepted August 2, 2006; Published September 15, 2006)

\begin{abstract}
We investigated the dielectric normal mode relaxation in dilute and semidilute solutions of poly(propylene oxide)(PPO) and poly(butylene oxide)(PBO) in benzene which is a good solvent for PPO but a marginal solvent for PBO. The results indicate that the normal mode relaxation times $\tau_{\mathrm{n}}$ in dilute solutions of PPO and PBO are proportional to $[\eta] M$ in agreement with the Rouse-Zimm theory where $[\eta]$ and $M$ denotes the intrinsic viscosity and the molecular weight, respectively. With increasing concentration $C, \tau_{\mathrm{n}}$ increases in proportion to $C[\eta]$. The dynamic crossover concentration between the dilute and semidilute regimes is about four times higher than the static crossover concentration $C^{*}$. The relaxation strength $\Delta \varepsilon$ divided by $C$ decreases with increasing concentration indicating that the mean square end-to-end distance $\left\langle r^{2}\right\rangle$ decreases on account of the screening of the excluded volume effect. The concentration dependence of $\left\langle r^{2}\right\rangle$ agrees approximately with the scaling theory. [doi:10.1295/polymj.PJ2006063] KEY WORDS Dielectric Normal Mode Relaxation / Dilute Solution / Semidilute Solution / Poly(propylene oxide) / Poly(butylene oxide) / End-to-end Distance /
\end{abstract}

Dielectric normal mode relaxation spectroscopy has been widely used to examine dynamics of polymer chains associated with fluctuation of the end-to-end vector. ${ }^{1-3}$ This method also provides fruitful information on the mean square end-to-end distance., ${ }^{2,3}$ Among various Type-A polymers exhibiting dielectric normal mode relaxation, poly(propylene oxide) (PPO) has been studied long after the pioneering studies reported by Stockmayer and his coworkers. ${ }^{1,4-8}$ Those studies were made for bulk PPO with low molecular weight (MW). The dielectric normal mode relaxations of the other bulk polyethers including poly(butylene oxide) (PBO) have been reported recently. ${ }^{9-11}$ However, no dielectric data of polyethers in the solution state have been reported.

Although many dielectric studies of the normal mode relaxation have been reported for bulk polymers, relatively small numbers of the studies on the normal mode relaxation in solution have been reported for polyisoprene, ${ }^{12-14} \operatorname{poly}\left(\varepsilon\right.$-caprolactone)(PCL),${ }^{15,16}$ poly(varelolactone), ${ }^{16}$ and others. ${ }^{17-19}$ In those studies, the effects of MW, concentration $C$, and solvent quality on the average relaxation time $\tau_{\mathrm{n}}$, the relaxation spectrum, and relaxation strength $\Delta \varepsilon$ were investigated. In this article we report the dielectric normal mode relaxations of PPO and $\mathrm{PBO}$ with high MW in dilute and semidilute solutions in benzene. Unfortunately the PPO samples used in this study had relatively broad distribution of MW. Obviously the dielectric data for such a sample are not suited for the analyses of the relaxation spectra. Therefore we will minimize the discussion on the width of the loss curves. However as described below $\tau_{\mathrm{n}}$ and $\Delta \varepsilon$ are almost independent of the distribution of MW. The objective of this study is to examine the $C$ and MW dependences of $\tau_{\mathrm{n}}$ and $\Delta \varepsilon$ of PPO and PBO solutions in the dilute and semidilute regimes.

\section{EXPERIMENTAL}

\section{Material}

Samples of PPO were prepared by polymerization of propylene oxide with $\mathrm{Zn}\left(\mathrm{CH}_{2} \mathrm{CH}_{3}\right)_{2}$ and trace amount of water following the conditions reported by Booth et al. ${ }^{20}$ Thus prepared PPO samples had very broad MW distribution and they were fractionated in a mixed solvent of dimethylformamide and water in the temperature range from 345 to $350 \mathrm{~K}$. The reason for the fractionation at high temperatures was that PPO crystallized in the precipitated phase below $340 \mathrm{~K}$ which prevented successful fractionation. Due to this difficult condition, the fractionated samples had still relatively broad distribution. Samples of PBO were prepared from butylene oxide with potassium hydride as the catalyst. ${ }^{21}$ The details of preparation and characterization of PBO were reported previously. ${ }^{10}$

The absolute values of the weight average molecular weight $M_{\mathrm{w}}$ were measured by a GPC (Tosoh LS-8000) equipped with detectors of light scattering (LS) and differential refractive index (RI). The elution

${ }^{\dagger}$ To whom correspondence should be addressed (Tel/Fax: +81-6-6848-9265, E-mail: k-adachi@tcct.zaq.ne.jp). 
Table I. Sample characteristics

\begin{tabular}{lrccc}
\hline \multicolumn{1}{c}{ code } & $10^{-3} M_{\mathrm{w}}$ & $M_{\mathrm{w}} / M_{\mathrm{n}}$ & {$[\eta] / \mathrm{mL} \mathrm{g}^{-1}$} & $10^{2} C^{*} / \mathrm{g} \mathrm{mL}^{-1}$ \\
\hline PPO-56 & 5.6 & 1.55 & 50.7 & 1.97 \\
PPO-90 & 9.0 & 1.66 & 73.1 & 1.36 \\
PPO-110 & 11.0 & 1.54 & 85.3 & 1.17 \\
PPO-171 & 17.1 & 1.28 & 120 & 0.83 \\
PPO-199 & 19.9 & 1.93 & 135 & 0.74 \\
& & & & \\
PBO-47 & 4.7 & 1.20 & 46.0 & 2.17 \\
PBO-51 & 5.1 & 1.36 & 48.9 & 2.04 \\
PBO-55 & 5.5 & 1.30 & 52.9 & 1.89 \\
PBO-95 & 9.5 & 1.19 & 72.4 & 1.38 \\
\hline
\end{tabular}

volume $\left(\mathrm{EV}_{\max }\right)$ at which the signal from the RI detector became maximum was proportional to $\log M_{\mathrm{w}}$. The polydispersity $M_{\mathrm{w}} / M_{\mathrm{n}}$ was determined from the calibration curve of $\log M$ versus $\mathrm{EV}_{\max }$ with approximation of $M=M_{\mathrm{w}}$. The sample characteristics are listed in Table I. The GPC curves measured by the RI detector exhibited asymmetrical shapes with a broader distribution in the low MW side. However the RI curve was approximately symmetrical in the peak region around the $\mathrm{EV}_{\max }$. The theoretical $M_{\mathrm{w}}$ and $M_{\mathrm{w}} / M_{\mathrm{n}}$ for the case where the weight fraction $w$ of the component with $\mathrm{MW}=M$ is given by the Gaussian distribution are discussed in the Appendix.

\section{Method}

Dielectric measurements were performed by using a transformer bridge (General Radio, model GR-1615, USA) and a RLC meter (QuadTech, model 7600, USA). Dielectric cell for measurements of solution was reported previously. ${ }^{22}$ Dielectric measurements were made at $300 \mathrm{~K}$ for solutions with polymer concentrations from 1 to $20 \mathrm{wt} \%$. Concentration $C$ of solutions in the unit of $\mathrm{g} \mathrm{mL}^{-1}$ was calculated from the weight fraction of the components by assuming the additivity of the densities. Here the densities of PPO, PBO, and benzene are reported to be 1.01, 0.98 , and $0.77 \mathrm{~g} \mathrm{~mL}^{-1}$, respectively. ${ }^{8,23}$ The intrinsic viscosity was measured with a Ubbelohde viscometer at $300 \mathrm{~K}$.

\section{RESULTS AND DISCUSSION}

\section{Intrinsic Viscosity}

The intrinsic viscosity $[\eta]$ is an important quantity for analyses of solution properties. Although $[\eta]$ for $\mathrm{PPO} /$ benzene solution was reported by Allen et al. ${ }^{24}$ that for $\mathrm{PBO} /$ benzene solution is not available in literature. Measurements of $[\eta]$ were carried out at $300 \mathrm{~K}$ and $[\eta]$ for the samples of PBO were determined as listed in Table I. Those data are fitted to the Mark-Houwink-Sakurada equation, $[\eta]=K_{\eta} M_{\mathrm{w}}{ }^{\alpha}$, with $K_{\eta}=0.0588$ and $\alpha=0.62$. The values of $[\eta]$

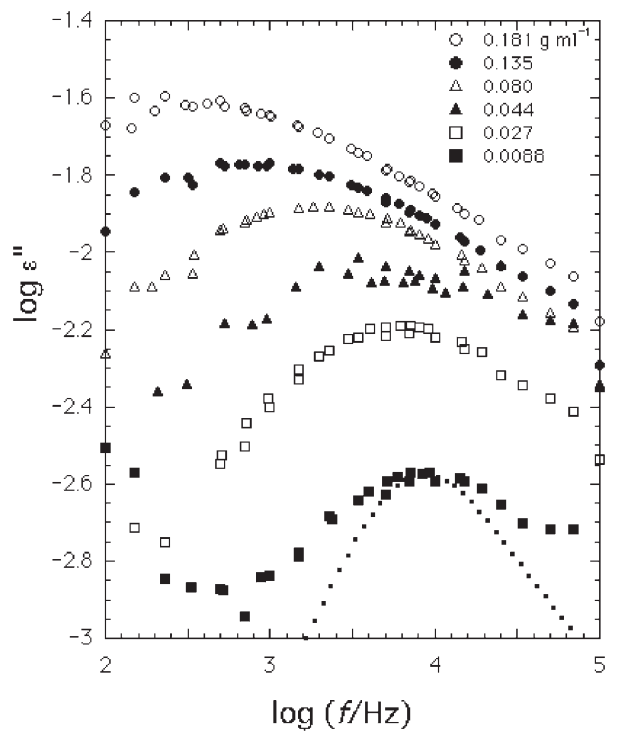

Figure 1. Double logarithmic plots of $\varepsilon^{\prime \prime} v s$. frequency $f$ for solutions of PPO-199. The concentrations are indicated in the figure. The dotted line shows the $\varepsilon^{\prime \prime}$ curve calculated with eqs 1 and 2 .

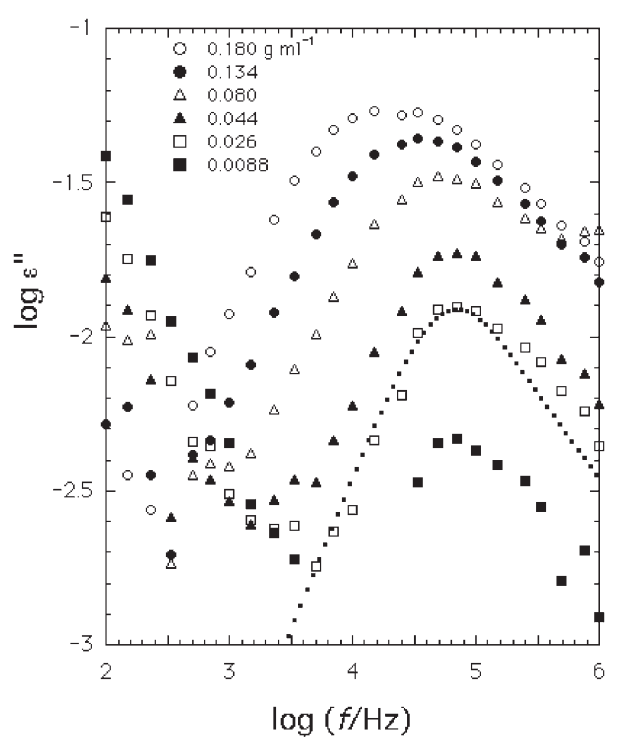

Figure 2. Double logarithmic plots of $\varepsilon^{\prime \prime} v s$. frequency $f$ for solutions of PBO-95. The dotted line shows the $\varepsilon^{\prime \prime}$ curve calculated with eqs 1 and 2.

and the overlapping concentration $C^{*}(\approx 1 /[\eta])^{25}$ are listed in Table I.

\section{Dielectric Relaxation in $\mathrm{PPO}$ and $\mathrm{PBO}$ Solutions}

Figures 1 and 2 show the representative frequency $f$ dependences of the dielectric loss factor $\varepsilon^{\prime \prime}$ in benzene solutions of PPO-199 and PBO-95, respectively. Solutions of the other PPO and PBO samples exhibited similar behavior. It was observed that the loss maximum frequency $f_{\mathrm{m}}$ shifted to high frequencies with decreasing MW if compared at the same $C$. This indicates that the observed dielectric relaxation is due to 
the normal mode process. It is seen that in the range of $C<0.03 \mathrm{~g} \mathrm{~mL}^{-1}$ the loss maximum frequencies $f_{\mathrm{m}}$ for PPO-199 are almost independent of concentration and stay at the same frequency although the intensity of loss peak increases in proportion to $C$. For PBO-95 solutions similar behavior is seen in the range of $C<0.05 \mathrm{~g} \mathrm{~mL}^{-1}$. This regime is defined as the dynamically dilute regime. The dilute regime shifts to lower frequency side with increasing MW. Obviously the decrease of $f_{\mathrm{m}}$ with increasing $C$ in the semidilute regime is due to entanglement effects and will be discussed later.

The complex dielectric constant $\varepsilon^{*}\left(=\varepsilon^{\prime}-i \varepsilon^{\prime \prime}\right)$ of type-A polymers is given theoretically by the FourierLaplace transform of the autocorrelation function $\langle\boldsymbol{r}(0) \cdot \boldsymbol{r}(t)\rangle$ of the end-to-end vector $\boldsymbol{r}(t)$ where $t$ is time. The bead-spring model has been used to express the MW dependence of $\langle\boldsymbol{r}(0) \cdot \boldsymbol{r}(t)\rangle .^{26,27}$ Thus $\varepsilon^{*}$ of dilute solutions is expressed as ${ }^{27}$

$$
\begin{aligned}
& \frac{\varepsilon^{*}(\omega)-\varepsilon_{\infty}}{\Delta \varepsilon}=\frac{8}{\pi^{2}} \sum_{p: o d d}^{\infty} \frac{1}{p^{2}\left(1+i \omega \tau_{p}\right)} \\
& \tau_{p}=\frac{\pi^{3 / 2} \eta_{s} N^{3 / 2} b^{2}}{12^{1 / 2} k_{B} T \lambda_{p}}
\end{aligned}
$$

where $\varepsilon_{\infty}$ is the unrelaxed dielectric constant, $\omega$ the angular frequency, $\Delta \varepsilon$ the relaxation strength, $\tau_{\mathrm{p}}$ the relaxation time for the $p$-th normal mode, $\eta_{\mathrm{s}}$ the solvent viscosity, $N$ the number of the beads, $b$ the average distance between the beads; $k_{\mathrm{B}} T$, the thermal energy; and $\lambda_{\mathrm{p}}$, the $p$-th eigenvalue tabulated by Zimm et al. ${ }^{28}$

The dotted curves in Figures 1 and 2 indicate the theoretical $\varepsilon^{\prime \prime}$ curves calculated with the Zimm theory (eqs 1 and 2). ${ }^{27}$ The peak positions of the theoretical $\varepsilon^{\prime \prime}$ curves are shifted so that they coincide with those of the experimental $\varepsilon^{\prime \prime}$ curve. It is seen that the experimental $\varepsilon^{\prime \prime}$ curves in the dilute regime are broader than the theoretical curves especially in the $\varepsilon^{\prime \prime}$ curve of PPO. Previously it was found that the $\varepsilon^{\prime \prime}$ curves in dilute solution conform approximately to eqs 1 and $2 .{ }^{14,16}$ The deviation of the present data of dilute solution from the Zimm theory can be attributed to a broader distribution of MW than used previously $\left(M_{\mathrm{w}} / M_{\mathrm{n}}<1.1\right)$. We also note in Figures 1 and 2 that the loss curves broaden with increasing $C$. Similar behavior was observed previously for semidilute solutions of polyisoprene, $\operatorname{poly}(\varepsilon$-caprolactone), poly(varelolacton), and poly(DL-lactic acid). ${ }^{17-17}$ In the present solutions, the broad $\varepsilon^{\prime \prime}$ curves in semidilute solutions are due to both the entanglement effects and the MW distribution.

Above results indicate that in order to analyze the data quantitatively, it is necessary to examine the effect of MW distribution on the $\varepsilon^{\prime \prime}$ curve. The theo- retical $\varepsilon^{\prime \prime}$ curves for samples with MW distribution are calculated in the Appendix by assuming that the MW dependence of weight fraction $w$ of components is Gaussian. The results indicate that although the broadness increases with the distribution of $\mathrm{MW}, f_{\mathrm{m}}$ is almost independent of the MW distribution when the MW $\left(M_{0}\right)$ at which $w$ becomes maximum is fixed. As discussed in the experimental section, $M_{0}$ defined in the Appendix corresponds to $M_{\mathrm{w}}$ of our samples due to a broader distribution of $w$ versus $\log M$ in the low MW side. Therefore we will focus on the $M_{\mathrm{w}}$ dependence of $\tau_{\mathrm{n}}\left(=1 / 2 \pi f_{\mathrm{m}}\right)$ in the following sections.

\section{Relaxation Time in Dilute to Semidilute Regime}

In the present study we carried out dielectric measurements on solutions with concentration above $1 \mathrm{wt} \%$. For solutions of some samples (PPO-171 and PPO-110) measurements were made only at $5 \mathrm{wt} \%$. For analyses of the relaxation time $\tau_{\mathrm{n}}$ in the dilute regime, it is needed to reduce the data of $\tau_{\mathrm{n}}$ to those $\tau_{\mathrm{n} 0}$ at infinite dilution. For this purpose we utilized the empirical relation on the $\log \tau_{\mathrm{n}}$ versus $C$ plots which was used by Jones et al. ${ }^{15,29}$

$$
\tau_{1}=\tau_{1 o} \exp \left(C A^{\prime}[\eta]\right)
$$

where $A^{\prime}$ is the constant. This equation indicates that the logarithm of dielectric relaxation time $\tau_{\mathrm{n}}$ is proportional to $C$ in dilute and semidilute solutions. Muthukumar and Freed proposed that the $p$-th relaxation time $\tau_{\mathrm{p}}$ of the Zimm theory depends on $C$ as given by ${ }^{30,31}$

$$
\begin{aligned}
\tau_{p}= & \tau_{p o}\left[1+C A p^{-\kappa}-2^{0.5}\left(C A p^{-\kappa}\right)^{1.5}\right. \\
& \left.+2\left(C A p^{-\kappa}\right)^{2.0}+\cdots\right]
\end{aligned}
$$

where $\tau_{\mathrm{p} 0}$ is the $p$-th relaxation time at infinite dilution; $A$, the constant proportional to the intrinsic viscosity $[\eta]$, and $\kappa$ is equal to $3 v-1 .{ }^{32}$ Here $v$ is the Flory exponent of the MW dependence of the chain dimension. ${ }^{32}$ Since eq 4 resembles to the expansion of eq 3 , the longest relaxation time $\tau_{1}(p=1)$ given by eq 4 is approximately equivalent to eq 3 .

Figures $3 \mathrm{a}$ and $3 \mathrm{~b}$ show the $\log \tau_{\mathrm{n}}$ versus $C[\eta]$ plots for PPO and PBO solutions. We see that the plots are approximately straight line and conform to eq 3 . From the slope of the plots, $A^{\prime}$ is determined to be $0.16 \pm 0.01$ for both of the $\mathrm{PPO} /$ benzene and $\mathrm{PBO} /$ benzene systems. Previously we found for semidilute polyisoprene solutions that $A^{\prime}=0.29$, irrespective of the MW and solvent quality. ${ }^{12-14}$ We also reported that $A^{\prime}$ for semidilute solutions of poly $(\varepsilon$-caprolactone) (PCL), poly(varelo lactone), and poly(DL-lactic acid) (PLA) in benzene were 0.26, 0.21 and 0.24, respectively. ${ }^{16,17}$ Jones et al. ${ }^{15}$ reported $A^{\prime}=0.21$ for 


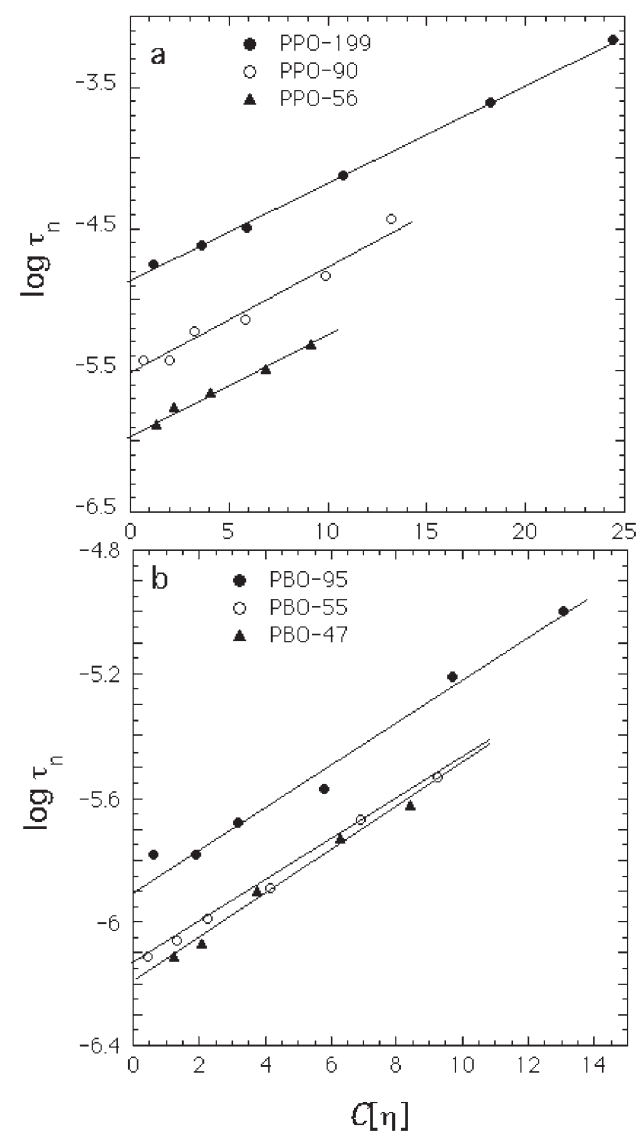

Figure 3. Plots of $\log \tau_{\mathrm{n}}$ with respect to $C[\eta]$ for solutions of PPO (a) and for those of PBO (b).

dioxane solutions of PCL. Baysal and Stockmayer ${ }^{34}$ reported for ternary solutions of PCL/poly(chlorostyrene)/dioxane that $A^{\prime}=0.35$. The value of $A^{\prime}$ for present PPO and PBO solutions are smaller than those of the other polymers. It appears that $A^{\prime}$ depends on the chemical structure of polymers.

By extrapolating the $\log \tau_{\mathrm{n}} v s . C[\eta]$ plots to zero concentration as shown in Figure 3, we determined the relaxation time $\tau_{\mathrm{n} 0}$ at infinite dilution for PPO and PBO solutions. For PPO-171 and PPO-110, we carried out dielectric measurements only on $5 \mathrm{wt} \%$ solutions. For these solutions, $\tau_{\mathrm{n} 0}$ was estimated by drawing straight lines with $A^{\prime}=0.16$. On account of scattering of the data points, the error in the determination of $\log \tau_{\mathrm{n} 0}$ is estimated to be \pm 0.1 . The MW dependence of $\tau_{\mathrm{n} 0}$ thus determined are shown in Figure 4 in which MW at which the GPC (RI) signal becomes maximum is used as the abscissa to avoid the influence of the polydispersity of MW. As shown in the Appendix, $f_{\mathrm{m}}$ is almost independent of the MW distribution if $M_{0}$ is used as the average MW and as described in the experimental section $M_{\mathrm{w}}$ corresponds to $M_{0}$.

In order to compare the results shown in Figure 4 with the Zimm theory which is applicable only to $\theta$ solutions (eqs 1 and 2), the theory has to be modified

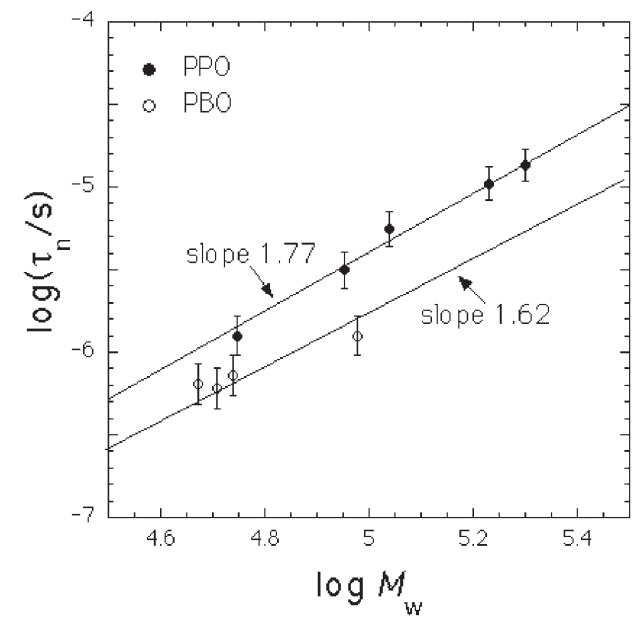

Figure 4. Molecular weight dependence of the normal mode relaxation time at infinite dilution $\tau_{\mathrm{n} 0}$. The slopes of the straight lines for the data of PPO and PBO solutions are 1.77 and 1.62, respectively.

so that it is applicable to solutions in good and marginal solvents. It is reminded that benzene is a good solvent for PPO and a marginal solvent for PBO as seen from the exponents $\alpha$ of the Mark-HouwinkSakurada equation: $\alpha=0.77$ for PPO/benzene and $\alpha=0.62$ for $\mathrm{PBO} /$ benzene solutions. Eq 2 is rewritten as: ${ }^{27}$

$$
\tau_{1}=K \frac{M \eta_{s}[\eta]}{R T}
$$

where $K$ is the constant and $\eta_{\mathrm{s}}$ the solvent viscosity. The Rouse theory ${ }^{26}$ also predicts eq 5 . The values of $K$ for the free draining and non-draining models are 1.22 and 0.85 , respectively. ${ }^{26,27}$ Returning to Figure 4 we see that the slopes of the $\log \tau_{\mathrm{n} 0}$ versus $\log M_{\mathrm{w}}$ plots for PPO and PBO are approximately 1.9 and 1.2, respectively. On the other hand eq 5 predicts the slopes of 1.77 and 1.62 for PPO and PBO solutions, respectively. We consider that the disagreements are due to experimental error in determination of $\tau_{\mathrm{n} 0}$. The slopes of the straight lines for PPO and PBO solutions are 1.77 and 1.62, respectively. We see that the experimental data points conform to eq 5 within the experimental error.

Figure 5 shows double logarithmic plots of $\tau_{\mathrm{n} 0}$ with respect to $M_{0} \eta_{\mathrm{s}}[\eta] / R T$ for dilute solutions of $\mathrm{PPO}$ and PBO. ${ }^{3}$ We see that all plots conform approximately to a universal straight line of the slope of unity. Although the slope of the plots includes a relatively high error as discussed above, the absolute values of $\tau_{\mathrm{n} 0}$ is close to the theory. The front factors $K$ for solutions of PPO and PBO become 1.90 and 1.00, respectively. It was reported that the average value of the experimental $K$ is $1.4 \pm 0.5$ for dilute solutions of various typeA polymers. ${ }^{3}$ The $K$ value of PPO solutions is slightly larger than the average value but that of $\mathrm{PBO}$ solution 


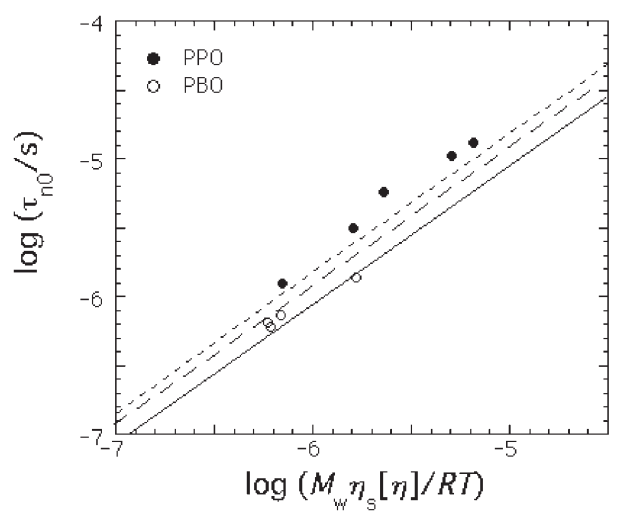

Figure 5. Double logarithmic plots of $\tau_{\mathrm{n} 0}$ vs. $M_{\mathrm{w}} \eta_{\mathrm{s}}[\eta] / R T$. The solid-, dashed- and dotted lines represent the Zimm theory, Rouse theory, and experimental average for solutions of various polymers. $^{3}$

is close to the Zimm theory. The previous data indicate that the slight deviation of the experimental $K$ values from the Zimm theory does not depend on the solvent quality but depends on the chemical characteristics of the polymer and solvent. The origin of the deviation of the $K$ value from the theory is an issue to be clarified in future.

\section{Effect of Entanglement on the Longest Relaxation Time}

In the previous section we analyzed the concentration dependence of the relaxation times of PPO and PBO solutions in the crossover concentration regime in terms of the $\log \tau_{\mathrm{n}} v s C$ plots (eq 3 ). In order to examine the crossover from the dilute regime to the entanglement regime, the double logarithmic plot of $\tau_{\mathrm{n}}$ vs $C$ is effective as shown in Figures 6a and 6b. Although eq 3 indicates that $\log \tau$ changes in proportion to $C$ in the crossover regime, the change is small over the scale of $\log C$ and therefore $\log \tau_{\mathrm{n}}$ is almost independent of $\log \mathrm{C}$, but in the semidilute and concentrated regimes, $\log \tau_{\mathrm{n}}$ increases with $\log C$.

In order to determine the dynamic crossover concentration $C_{\tau}{ }^{*}$ we employ the theory proposed by de Gennes who developed the reptation theory in semidilute solutions assuming that the correlation length $\xi$ of the segment density is equal to the diameter of the tube in which chains are constrained. ${ }^{35}$ The longest relaxation time $\tau_{\mathrm{d}}$ (tube disengagement time for the non-draining model) is given by

$$
\tau_{d} \propto \zeta N^{3}\left(C / C^{*}\right)^{(3-3 v) /(3 v-1)}
$$

where $\zeta$ is the friction coefficient, $\mathrm{N}$ the number of beads, and $v$ the excluded volume exponent. This equation predicts that $\log \tau_{\mathrm{n}}$ is linear with respect to $\log C$ with the slope of $(3-3 v) /(3 v-1)$ in the semidilute regime if the friction $\zeta$ is independent of $C$. It was found previously that $\zeta$ increases slightly with

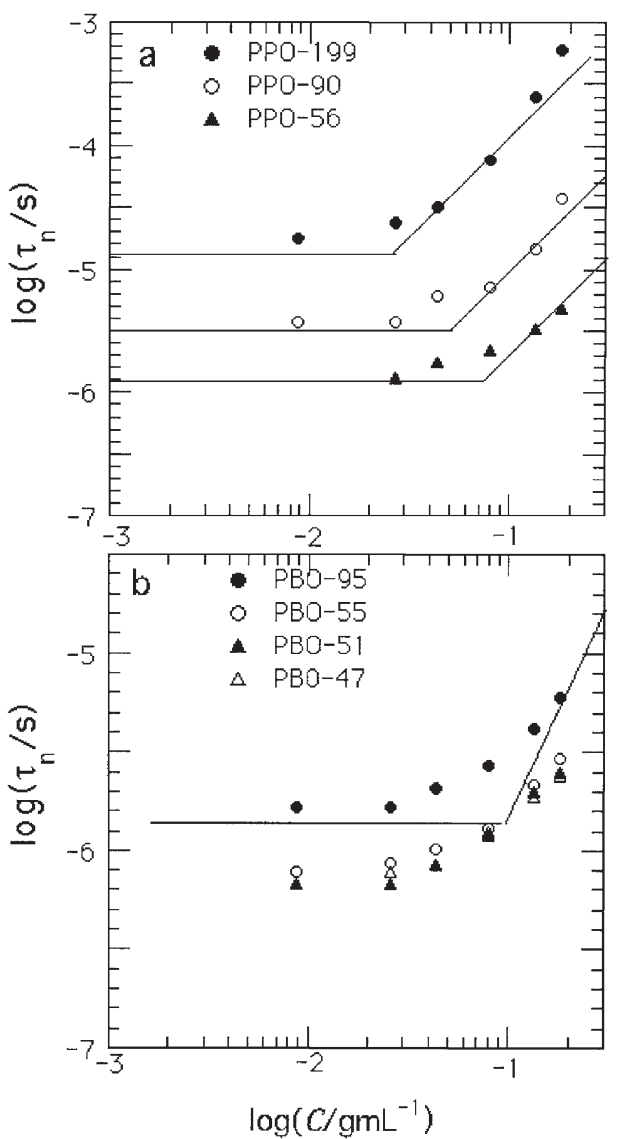

Figure 6. Double logarithmic plots of $\tau_{\mathrm{n}} v s$. $C$ for PPO solutions (a) and PBO solutions (b). The horizontal lines show the values of $\tau_{\mathrm{n} 0}$. The slopes of the lines in the high region are equal to $(3-3 v) /(3 v-1)$ where $v$ is the Flory parameter for the excluded volume effect.

increasing $C{ }^{12,17}$ We assume that the effect of $C$ on $\zeta$ in the crossover region is weak and we have drawn straight lines with the slope of $(3-3 v) /(3 v-1)$ as shown in Figures $6 \mathrm{a}$ and $6 \mathrm{~b}$. Here the values of $v$ for PPO and PBO in benzene are calculated to be 0.59 and 0.54 , respectively, from the exponent of the $[\eta] v s M$. Upward deviations of the plots from these straight lines in the high $C$ region of PPO solutions are due to the $C$ dependence of $\zeta$. As shown in Figure $6 \mathrm{a}, C_{\tau}{ }^{*}$ of PPO solutions is defined as the cross point of the extrapolated two straight lines in the dilute and semidilute regimes. The values of $C_{\tau}{ }^{*}$ for solutions of PPO-199, PPO-90, and PPO-56 are $0.026,0.050$, and $0.073 \pm 0.01 \mathrm{~g} \mathrm{~mL}^{-1}$, respectively.

For PBO solutions, eq 6 predicts a higher value of $(3-3 v) /(3 v-1)=2.23$ than that for PPO solutions (1.60). In the range of concentration where dielectric measurements were made, the slope of $\log \tau$ versus $\log C$ is less than 2.23 as shown in Figure 6b. This indicates that the highest concentration used in the measurements on $\mathrm{PBO}$ solutions is still less than the entangled regime in which eq 6 holds. The $\mathrm{C}$ dependences of $\log \tau$ observed in Figure $6 \mathrm{~b}$ are mostly 
responsible to the $\mathrm{C}$-dependence of the monomeric friction. Therefore $C_{\tau}{ }^{*}$ for PBO solutions cannot be determined uniquely on the basis of eq 6 . For PBO95 solutions we roughly estimate $C_{\tau}{ }^{*}$ to be $0.10 \pm$ $0.03 \mathrm{~g} \mathrm{~mL}^{-1}$ by drawing straight lines as shown in Figure $6 \mathrm{~b}$.

We note for PPO solutions that $C_{\tau}{ }^{*}$ is higher than the overlapping concentration $C^{*}(=1 /[\eta])$. The ratios of $C_{\tau}{ }^{*} / C^{*}$ for PPO-199, PPO-90, and PPO56 are 3.5, 3.7, and 3.7, respectively. These values are higher than those observed for solutions of polyisoprene and poly(lactic acid) in which $C_{\tau}{ }^{*} / C^{*}$ is 2 to $3 .^{12,17}$

Here we roughly estimate the MW between entanglements $M_{\mathrm{e}}$ in semidilute solutions from the characteristic molecular weight $M_{\mathrm{c}}{ }^{\mathrm{o}}\left(\approx 2 M_{\mathrm{e}}{ }^{\mathrm{o}}\right)$ in the undiluted state. Smith et al. reported $M_{\mathrm{c}}{ }^{\mathrm{o}}$ of bulk PPO to be $7000 .{ }^{36}$ Recently we estimated $M_{\mathrm{c}}{ }^{\circ}$ of PBO to be $6000 \pm 2000 .{ }^{10}$ It is well known that $M_{\mathrm{e}}$ is proportional to $1 / C$ in the concentrated regime of $C^{+}<C<\rho$, where $C^{+}$is the crossover concentration between the semidilute and concentrated regimes and $\rho$ is the density of the bulk state in the unit of $\mathrm{g} \mathrm{mL}^{-1}$. Since $C^{+}$is known to be 0.2 to 0.3 , we assume $C^{+}=$ 0.25 . In the semidilute regime of $C<C^{+}$, dynamic scaling model ${ }^{35}$ predicts that $M_{\mathrm{e}}$ is proportional to $\left(C / C^{*}\right)^{v /(1-3 v)}$. Thus we estimate $M_{\mathrm{e}}{ }^{\mathrm{o}}$ of PPO and PBO at $C=0.1$ to be 3500 and 3000 , respectively, and $M_{\mathrm{c}}$ of PPO and PBO at $C=0.1$ are estimated to be $57000 \pm 5000$ and $53000 \pm 5000$, respectively. The error range of \pm 5000 is due to the ambiguity of the value of $\mathrm{C}^{+}$. These results correspond well to the fact that $C_{\tau}{ }^{*}$ of solutions of PPO and PBO with $\mathrm{MW} \approx 50000$ is about $0.1 \mathrm{~g} \mathrm{~mL}^{-1}$.

\section{Relaxation Strength}

The dielectric relaxation strength $\Delta \varepsilon$ was determined from the Cole-Cole plots. The results of $\Delta \varepsilon / C$ for PPO and PBO solutions are shown in Figures $7 \mathrm{a}$ and $7 \mathrm{~b}$, respectively. As observed for solutions of polyisoprene, ${ }^{12-14}$ poly( $\varepsilon$-caprolactone) ${ }^{16}$ and poly(lactic acid), ${ }^{17}$ the relaxation strength decreases with increasing concentration due to the screening of the excluded volume effect on the chain dimension. The horizontal lines indicate the levels of $\Delta \varepsilon / \rho$ of the bulk PPO and PBO where $\rho$ is the bulk density. ${ }^{7,10}$ For type A polymers, $\Delta \varepsilon$ is proportional to the mean square end-to-end distance $\left\langle r^{2}\right\rangle / M$ :

$$
\frac{\Delta \varepsilon}{C}=\frac{4 \pi N_{A} \mu^{2}\left\langle r^{2}\right\rangle}{3 k_{B} T M} \quad \text { (in cgs esu unit) }
$$

where $\mu$ is the dipole moment per unit contour length and $\mathrm{N}_{\mathrm{A}}$ is the Avogadro constant. Here the internal field factor is assumed to be unity as discussed previously. ${ }^{2}$ Before the analyses of the experimental results, it is needed to examine the effect of MW distri-

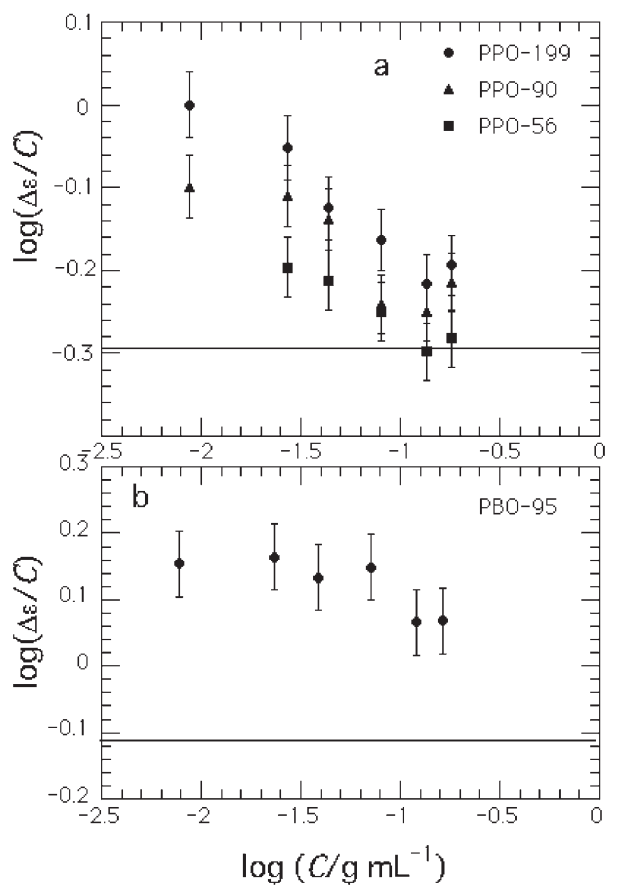

Figure 7. Double logarithmic plots of relaxation strength $\Delta \varepsilon$ divided by $C$ vs. $C$ for PPO solutions (a) and PBO solutions (b). The solid lines indicate the values of $\Delta \varepsilon$ divided by the density of bulk PPO and PBO.

bution on $\Delta \varepsilon / C$. This is done in the Appendix where it is shown that the effect is within the error of determination of $\Delta \varepsilon$ from the Cole-Cole plots.

The values of $\mu$ of PPO and PBO have been determined to be $1.10 \times 10^{-11}$ and $1.65 \times 10^{-11}$ esu $\left(3.67 \times 10^{-21}\right.$ and $\left.5.50 \times 10^{-21} \mathrm{C}\right)$, respectively. ${ }^{7,10}$ From these values the average end-to-end distances $\left\langle r^{2}\right\rangle^{0.5}$ of PPO-199, PPO-90, and PBO-95 in dilute benzene solution at $300 \mathrm{~K}$ become 51,31 , and $27 \mathrm{~nm}$, respectively. These values are longer than $\left\langle r^{2}\right\rangle^{0.5}$ in the unperturbed states 37,25 , and $21 \mathrm{~nm}$, respectively owing to the excluded volume effect: $\left\langle r^{2}\right\rangle \propto M^{2 v}$. Strictly speaking these values are based on the assumption that the characteristic ratio $C_{\infty}$ is independent of surrounding medium. If the potential energy of the internal rotation changes by the polymer-solvent interactions, the value of $C_{\infty}$ is required for each polymer/solvent system. However the dependence of $C_{\infty}$ on the solvent quality has not been clarified.

According to Daoud and Jannink, the $M_{\mathrm{w}}$ and concentration dependence of $\left\langle r^{2}\right\rangle$ in semidilute regime is given by $^{37}$

$$
\left\langle r^{2}\right\rangle \propto C^{(2 v-1) /(1-3 v)}
$$

This equation predicts that the slope of the $\log (\Delta \varepsilon / C)$ vs $\log C$ in the semidilute regimes of PPO and PBO solutions are -0.23 and -0.13 , respectively. The slopes of the plots shown in Figures $7 \mathrm{a}$ and $7 \mathrm{~b}$ approximately agree with the theory. 


\section{SUMMARY}

We have examined the dielectric normal mode relaxation in benzene solutions of PPO and PBO. The excluded volume parameter $v$ is determined to be 0.59 and 0.54 for PPO and PBO solutions, respectively, from the molecular weight (MW) dependence of the intrinsic viscosity $[\eta]$. The logarithm of the normal mode relaxation time $\tau_{\mathrm{n}}$ is found to be proportional to concentration $C$ (in $\mathrm{g} \mathrm{mL}^{-1}$ ). The slope $A^{\prime}$ of the $\log \tau_{\mathrm{n}} v s C[\eta]$ is 0.16 for both of PPO and PBO solutions which is smaller than the $A^{\prime}$ values for semidilute solutions of the other polymers. The dielectric relaxation time $\tau_{\mathrm{n} 0}$ at infinite dilution has been determined by extrapolating the $\log \tau_{\mathrm{n}}$ versus $C$ plots. The double logarithmic plots of $\tau_{\mathrm{n} 0}$ versus MW is linear and the slopes are found to be 1.77 and 1.62 for the PPO and PBO solutions, respectively. These slopes are in agreement with the theoretical slopes for the relationship of $\tau_{\mathrm{n} 0} \propto M[\eta]$ predicted by the RouseZimm theory. The plots of $\tau_{\mathrm{n} 0} v s . M[\eta] \eta_{\mathrm{s}} / R T$ conform approximately to the universal plots where $\eta_{\mathrm{s}}$ is the solvent viscosity. The dynamical crossover concentration $C_{\tau}{ }^{*}$ has been determined by the $\log \tau_{\mathrm{n}} v s . \log C$ plots. $C_{\tau}{ }^{*}$ is $c a 2$ to 3 times higher than the overlapping concentration $C^{*}$ and decreases with increasing MW. The relaxation strength $\Delta \varepsilon / C$ versus $C$ plots exhibits the dilute and semidilute regimes. In the dilute regime the end-to-end distance of PPO-199 and PBO-95 are 51 and $29 \mathrm{~nm}$, respectively. The slopes of the $\log \Delta \varepsilon / C$ vs. $\log C$ in the semidilute regimes of $\mathrm{PPO}$ and $\mathrm{PBO}$ solutions are -0.23 and -0.13 which agree with the scaling theory.

Acknowledgment. We thank Professor N. Ueyama of Osaka University for his valuable advises in synthesis of PPO.

\section{APPENDIX}

Based on the Zimm theory (eqs 1 and 2), we examine the effect of the distribution of MW on the $\varepsilon^{\prime \prime}$ curve for the case that the weight fraction $w$ of the molecules with $M$ is given by the Gaussian distribution with respect to $\ln M$ :

$$
w(\ln M)=\kappa \exp \left[-\beta^{2}\left(\ln M-\ln M_{0}\right)^{2}\right]
$$

where $\kappa$ is the constant, $\beta$ the parameter representing the broadness of the distribution, and $M_{0}$ the MW at which $w$ becomes maximum. The values of $M_{\mathrm{w}} / M_{\mathrm{n}}$ and $M_{\mathrm{w}}$ are given by

$$
\begin{aligned}
& M_{\mathrm{w}} / M_{\mathrm{n}}=\exp \left(\beta^{-2} / 2\right) \\
& M_{\mathrm{w}}=M_{0} \exp \left(3 \beta^{-2} / 4\right)
\end{aligned}
$$

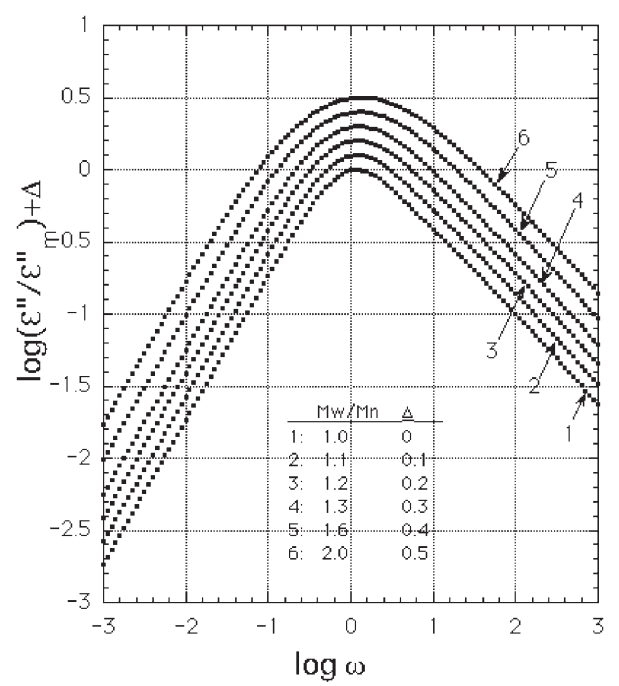

Figure A-1. The $M_{\mathrm{w}} / M_{\mathrm{n}}$ dependence of theoretical $\varepsilon^{\prime \prime}$ curve under the condition of fixed $M_{0}$. The curves $1-6$ corresponds to $M_{\mathrm{W}} / M_{\mathrm{n}}$ of 1.0 to 2.0 and each curve is shifted by $\Delta$ as indicated in the figure.

The $\varepsilon^{\prime \prime}$ curves are calculated numerically for the cases that $\tau_{1}$ of the Zimm theory at $M=M_{0}$ is unity.

Figure A. 1 shows the $M_{\mathrm{w}} / M_{\mathrm{n}}$ dependence of the normalized $\log \varepsilon^{\prime \prime}$ curves for the case of fixed $M_{0}$. It is seen that the frequency of the loss maximum shifts slightly to high frequency side with increasing $M_{\mathrm{w}} / M_{\mathrm{n}}$. This is due to the higher order modes given by eq 2 .

Now we examine the effect of the distribution of MW on the relaxation strength $\Delta \varepsilon$. From eq 7 , $\Delta \varepsilon / C$ is given by $\xi M^{2 v-1}$ where $\xi$ is constant. When the distribution of $\mathrm{MW}$ is given by eq $\mathrm{A} \cdot 1, \Delta \varepsilon / C$ is given by

$$
\frac{\Delta \varepsilon}{C}=\frac{\int_{0}^{\infty} \kappa \xi M^{2 v-1} \exp \left[-\beta\left(\ln M-\ln M_{0}\right)^{2}\right] d M}{\int_{0}^{\infty} \kappa \exp \left[-\beta\left(\ln M-\ln M_{0}\right)^{2}\right] d M}
$$

This equation is solved with the aid of eq A.2 as

$$
\frac{\Delta \varepsilon}{C}=\xi M_{0}^{2 v-1}\left(M_{\mathrm{w}} / M_{\mathrm{n}}\right)^{\left(4 v^{2}-1\right) / 2}
$$

This result indicates that in the case of a $\theta$ solution, $\Delta \varepsilon / C$ is independent of MW and MW distribution as expected. In the case of good solvent, $\Delta \varepsilon / C$ increases with increasing $M_{\mathrm{w}} / M_{\mathrm{n}}$. However the effect is small, i.e., in the case of PPO-199 solution for which $v=0.59$ and $M_{\mathrm{w}} / M_{\mathrm{n}}=1.93$, the value of $\log (\Delta \varepsilon / C)$ is larger than $\log \left(\xi M_{0}{ }^{2 v-1}\right)$ by 0.057 . This deviation is smaller than the other experimental error in determination of $\Delta \varepsilon$. Thus the effect of MW distribution on $\Delta \varepsilon / C$ is weak. 


\section{REFERENCES}

1. W. H. Stockmayer, Pure Appl. Chem., 15, 539 (1967).

2. K. Adachi and T. Kotaka, Prog. Polym. Sci., 18, 585 (1993).

3. K. Adachi, in "Dielectric Spectroscopy of Polymeric Materials," J. P. Runt and J. J. Fitzgerald, Ed., American Chemical Society, Washington, DC, 1997, Chapt. 9.

4. E. Baur and W. H. Stockmayer, J. Chem. Phys., 43, 4319 (1965).

5. J. Burke and W. H. Stockmayer, Macromolecules, 3, 647 (1969).

6. E. Schlösser and A. Schönhals, Prog. Colloid Polym. Sci., 91, 158 (1993).

7. T. Hayakawa and K. Adachi, Polymer, 42, 1724 (2001).

8. A. Kyritsis, P. Pissis, S.-M. Mai, and C. Booth, Macromolecules, 33, 4581 (2000).

9. R. Casalini and C. M. Roland, Macromolecules, 38, 1779 (2005).

10. M. Yamane, Y. Hirose, and K. Adachi, Macromolecules, 38, 9210 (2005).

11. M. Yamane, Y. Hirose, and K. Adachi, Macromolecules, 38, 10686 (2005).

12. K. Adachi and T. Kotaka, Macromolecules, 21, 157 (1988).

13. K. Adachi, Y. Imanishi, T. Shinkako, and T. Kotaka, Macromolecules, 22, 2391 (1989).

14. O. Urakawa, K. Adachi, and T. Kotaka, Macromolecules, 27, 7410 (1994).

15. A. A. Jones, W. H. Stockmayer, and R. J. Molinari, J. Polym. Sci., Polym. Symp., 54, 227 (1976).

16. O. Urakawa, K. Adachi, T. Kotaka, Y. Takemoto, and H. Yasuda, Macromolecules, 27, 7410 (1994).

17. J. Ren J, O. Urakawa, and K. Adachi, Polymer, 44, 847
(2003).

18. S. Uzaki, K. Adachi, and T. Kotaka, Macromolecules, 21, 153 (1988).

19. K. Adachi and T. Kotaka, Macromolecules, 16, 1936 (1983).

20. C. Booth, Polymer, 5, 479 (1964).

21. A. Stolarzewich and D. Neugebauer, Macromol. Chem. Phys., 200, 2467 (1999).

22. Y. Imanishi, K. Adachi, and T. Kotaka, J. Chem. Phys., 89, 7585 (1988).

23. "Polymer Handbook," J. Brandrup and E. H. Immergut, Ed., John-Wiley, New York, 1989.

24. G. Allen, C. Booth, and M. M. Jones, Polymer, 5, 195 (1964).

25. P. G. de Gennes, "Scaling Concepts in Polymer Physics," Cornell Univ. Press, London, 1979.

26. P. E. Rouse, J. Chem. Phys., 21, 1272 (1953).

27. B. H. Zimm, J. Chem. Phys., 24, 269 (1956).

28. B. H. Zimm, G. L. Roe, and L. F. Epstein, J. Chem. Phys., 24, 279 (1956).

29. R. S. Spencer and J. L. Williams, J. Colloid Interface Sci., 2, 117 (1949).

30. M. Muthukumar and K. F. Freed, Macromolecules, 11, 843 (1978).

31. M. Muthukumar, Macromolecules, 17, 971 (1984).

32. P. J. Flory, "Principle of Polymer Chemistry," Cornell University Press, Ithaca, 1953.

33. K. Adachi and T. Kotaka, Macromolecules, 21, 157 (1988).

34. B. Baysal and W. H. Stockmayer, J. Mol. Liq., 56, 175 (1993).

35. P. G. de Gennes, Macromolecules, 9, 594 (1976).

36. B. A. Smith, E. T. Samulski, L. P. Yu, and M. A. Winnik, Macromolecules, 18, 1901 (1985).

37. M. Daoud and G. Jannink, J. Phys. (Paris), 37, 973 (1976). 